\title{
Solute transport and interface evolution in dissolutive wetting
}

\author{
JinHong Yang ${ }^{1,2}$, QuanZi Yuan ${ }^{1,2^{*}}$, and YaPu Zhao ${ }^{1,2^{*}}$ \\ ${ }^{1}$ State Key Laboratory of Nonlinear Mechanics, Institute of Mechanics, Chinese Academy of Sciences, Beijing 100190, China; \\ ${ }^{2}$ School of Engineering Science, University of Chinese Academy of Sciences, Beijing 100049, China
}

Received March 20, 2019; accepted May 7, 2019; published online August 12, 2019

\begin{abstract}
Dissolutive wetting, i.e., droplet wetting on dissolvable surfaces, is essential for various natural phenomena and industrial applications such as the formation of sinkholes, enhancing shale gas recovery, drug design, MEMS, and so on. It is difficult to predict the evolution of concentration field and solid-liquid interface owing to the coupled effects of wetting, diffusion, and convection. This study makes substantial progress by proposing a new theory based on Onsager's variational principle and finding two modes of solute transport, i.e., shifting and lifting modes. Furthermore, we investigate the influence of wetting and dissolution coupling on the interface shape using a phase diagram. Using our theory, we can predict and inversely predict the interface evolution.
\end{abstract}

wetting, diffusion, solid-liquid interface, concentration distribution

PACS number(s): $61.30 . \mathrm{Hn}, 68.35 . \mathrm{Fx}, 68.08 .-\mathrm{p}, 68.55 . \mathrm{Ln}$

Citation: J. H. Yang, Q. Z. Yuan, and Y. P. Zhao, Solute transport and interface evolution in dissolutive wetting, Sci. China-Phys. Mech. Astron. 62, 124611 (2019), https://doi.org/10.1007/s11433-019-9425-y

$\begin{array}{cl}\text { List of variables } \\ c & \text { Concentration } \\ c_{\mathrm{s}} & \text { Saturation concentration } \\ c_{0} & \text { The initial concentration near origin } \\ k_{\mathrm{B}} & \text { Boltzmann constant } \\ u_{\mathrm{c}} & \text { Characteristic convective velocity } \\ u_{\mathrm{f}} & \text { Height-averaged flow velocity } \\ v_{\text {mole }} & \text { Mole volume of the solute } \\ C_{a} & \text { Capillary number } \\ C_{n} & \text { Shape factor } \\ D & \text { Diffusion coefficient } \\ F & \text { Potential energy } \\ H_{\mathrm{a}} & \text { Concentration boundary-layer thickness } \\ H_{1} & \text { Lower height of the droplet } \\ H_{\mathrm{u}} & \text { Upper height of the droplet }\end{array}$

*Corresponding authors (QuanZi Yuan, email: yuanquanzi@lnm.imech.ac.cn; YaPu Zhao, email: yzhao@imech.ac.cn)
$L \quad$ Characteristic length

$N_{\mathrm{A}} \quad$ Avogadro number

$P e \quad$ Pélect number

$R \quad$ Droplet radius

$S \quad$ Spreading coefficient

$T$ Temperature

$V \quad$ Droplet volume

$\alpha \quad$ Convective intensity

$\phi \quad$ Mean volume concentration

$\gamma \quad$ Surface tension

$\eta \quad$ Solvent viscosity

$\theta \quad$ Contact angle

$\theta_{\text {le }} \quad$ Lower equilibrium contact angle

$\theta_{\text {ue }} \quad$ Upper equilibrium contact angle

$\tau_{\mathrm{D}} \quad$ Dissolution characteristic time

$\tau_{\mathrm{D}} \quad$ Diffusion characteristic time

$\xi$ Dissipation coefficient at the contact line 
$\Phi \quad$ Energy dissipation

$\Phi_{\eta} \quad$ Viscosity dissipation

$\Phi_{\xi} \quad$ Dissipation at the contact line

$\Omega \quad$ Surface area of a solute molecular

\section{Introduction}

Dissolutive wetting, i.e., droplet wetting on dissolvable surfaces, is a multidisciplinary area of research essential for natural and industrial phenomena on microscale to macroscale level $[1,2]$. It plays a significant role when acidic liquids are used to expand rock cracks for enhancing shale gas recovery [3], fabricating microelectromechanical systems and electronic devices via wet etching [4,5], and printing functional skin on material surfaces at the microscale level [6]. At the mesoscale level, dissolutive wetting plays an important role in the dissolution of drugs [7], controlling weld pool shape in welding, and erosion of materials $[8,9]$. The abovementioned examples involve the interactions between a fluid and dissolvable surface. In addition, this investigation can help in analyzing and predicting changes on planetary surfaces, such as the formation of sinkholes [10], karst topography [11], and scalloping morphology at macroscale level $[12,13]$. Despite its wide applications, there is a lack of suitable theoretical models to explain the phenomena of dissolutive wetting.

During the dissolutive wetting process, the droplet spreads over the dissolvable solid surface while the solute diffuses into the liquid (see Figure 1), unlike nondissolutive wetting $[2,14,15]$. While the solute diffuses into the droplet, the solid-liquid interface changes continuously [8]. However, the properties of the liquid depend on the distribution of the solutes, and the amount of solutes on the droplet surface determines the properties of the interface. Furthermore, chemical and surface tension gradients lead to internal convection in the droplet [16]. In addition, the dissolutive wetting process has two stages due to the difference in the relaxation times between wetting and diffusion $[17,18]$. Nevertheless, previous studies have primarily been proceeded by experiments $[19,20]$ and theoretical analyses have been inadequate [21-23] owing to the complex coupling of wetting, diffusion, and convection in dissolutive wetting. Previous studies have focused on the triple-phase contact line and evolution of the contact angle. Studies based on the interface shape and concentration field are lacking; however, predicting the interface shape and concentration field is key to the application of dissolutive wetting in industries.

Herein, we propose a new theory based on Onsager's variational principle and find two modes of solute transport. Other forms of solute transport are combinations of these two modes. Furthermore, this theory explains the influence of wetting and dissolution coupling on the interface shape via a phase diagram and can be used to predict the solid-liquid interface shape. We have conducted experiments to verify the existence of the two concentration evolution modes and to verify the correctness and reliability of the proposed model.
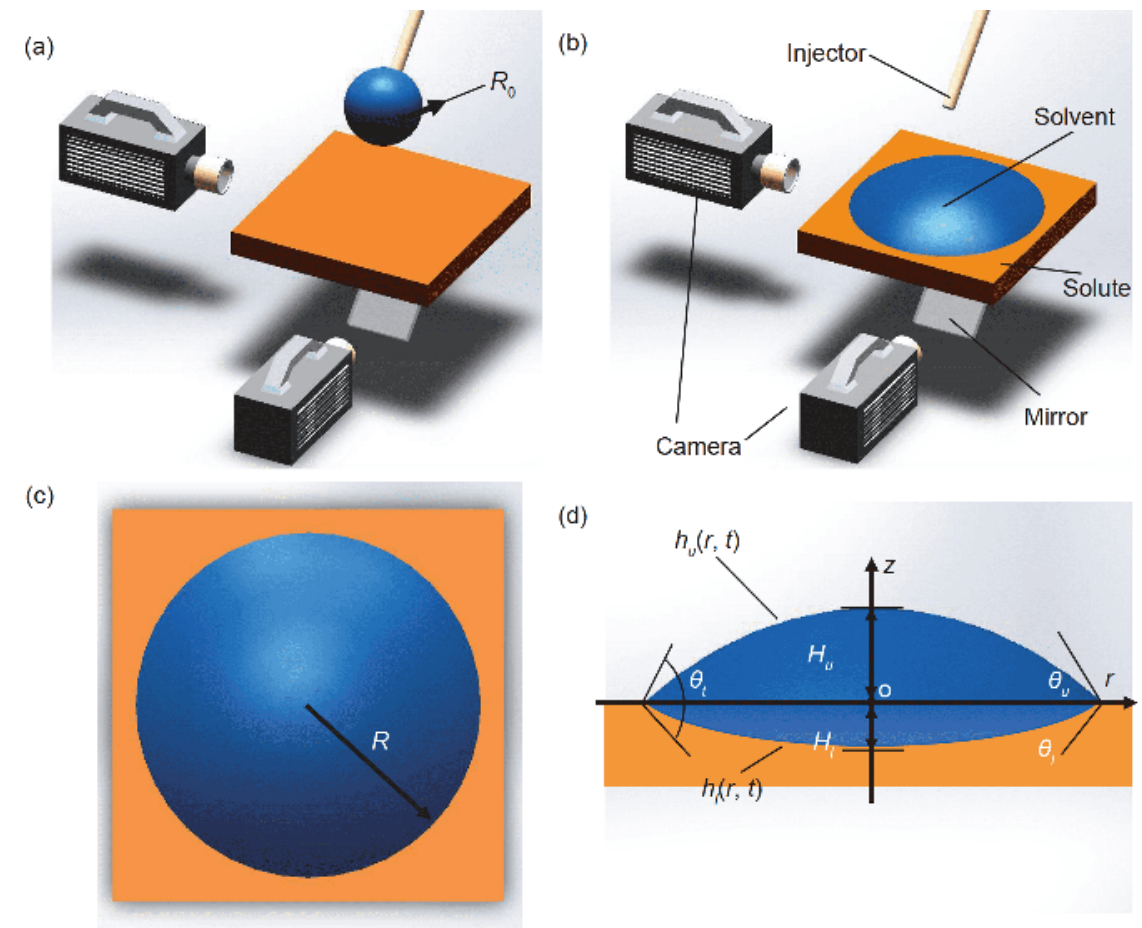

(d)

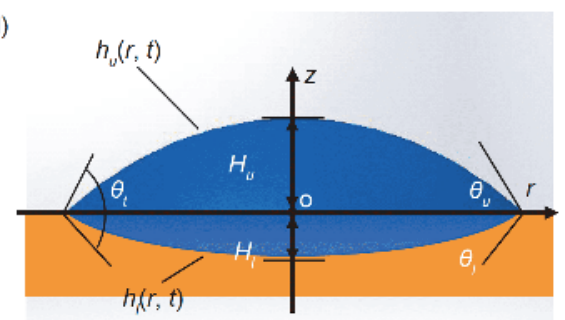

Figure 1 (Color online) Schematics of the model and the experimental setup. (a) In the initial state, a droplet with radius $R_{0}$ is produced by an injector. (b) In the transient state, the droplet spreads on the dissolvable substrate: the top (c) and side (d) views of the droplet. $H, R, \theta$, and $h$ are the instantaneous height, radius, contact angle, and height profile of the droplet, respectively. The subscripts $\mathrm{u}$ and 1 of all symbols represent upper and lower, respectively. 


\section{Experimental section}

We extract velocity information inside a droplet via particle image velocimetry (PIV), wherein particles with a diameter of $6 \mu \mathrm{m}$ are diluted to $0.004 \mathrm{wt} \%$ with a solvent. In our experiments, a solvent droplet with a volume of $1 \mu \mathrm{L}$ is deposited on a solid solute surface. The wetting properties of the droplet are investigated by conducting contact angle measurements. There are two types of solute substrate. One is made by heating a sugar solution comprising glucose and water (5:1 by mass) to $250^{\circ} \mathrm{C}$ and then casting it in a mold. The other is made using polyvinyl alcohol (PVA). We make a PVA thin layer via repeated suspension coating and drying; then the surface is treated with plasma. The fluorescence intensity is used to measure the concentration distribution. The mass ratio of dye powder, i.e., rhodamine $\mathrm{B}$, to solvent is $5 \times 10^{-6}$. The evolutions of the flow and concentration field in the droplet are recorded via an inverted fluorescence microscope. For PVA, in the concentration experiment, fluorescence at a concentration of $5 \times 10^{-6}$ by mass can also be added to the substrate. We remove the solvent using blotting paper and measure the shape of the hole via probe scanning (see Figure 3).

\section{Results and discussion}

The distribution of the flow field is the key variable. We compare the convective velocity and diffusion velocity using
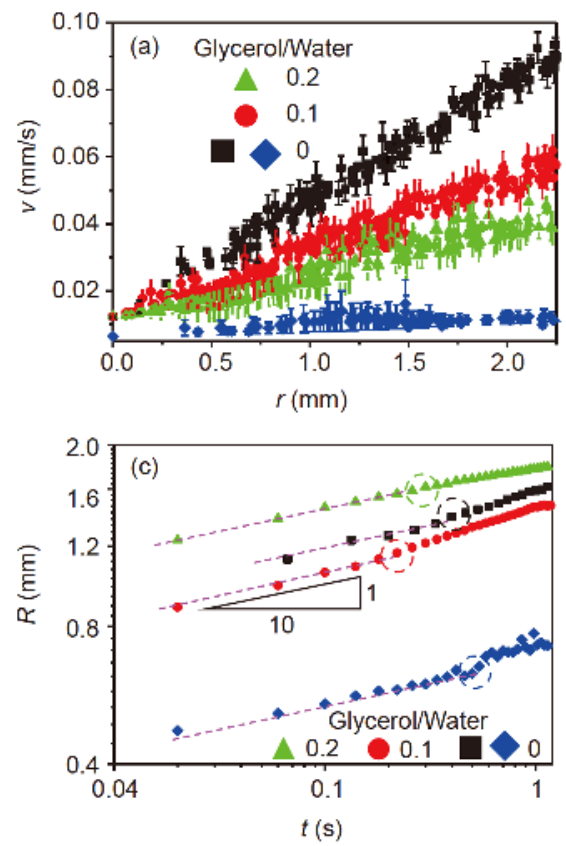

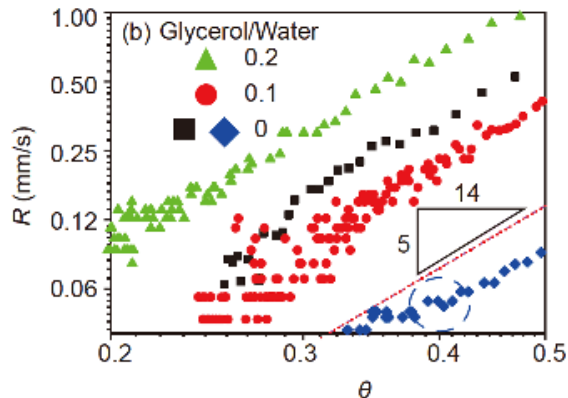

the Pélect number, $P e=u_{c} L / D$, where $u_{c}$ is the characteristic convective velocity, $L$ is characteristic length, and $D$ is diffusion coefficient. $\mathrm{Pe}$ is a combination of the Reynolds number and Schmidt number. Therefore, $P e$ relates to the flow state of the liquid and presents the comparison between viscous diffusion and mass diffusion. According to the PIV results of the typical dissolving couples, as shown in Figure 2(a), we find that $P e>1$ with a height-averaged velocity of the order of $10^{-5} \mathrm{~mm} \mathrm{~s}^{-1}$; therefore, the influence of convection on the mass transfer should be considered. In addition, a closer contact line indicates a faster convective speed, and height-averaged fluid velocity has a linear relation with the position along the $r$ direction. The positive velocity shows that the solute is transported toward the edge of the droplet. When the droplet volume is changing, the height-averaged flow velocity $\left(u_{\mathrm{f}}\right)$ can be written in the following form according to the results of the PIV experiments and ref. [24]:

$u_{\mathrm{f}}=\left(\frac{\dot{R}}{R}-\frac{\dot{V}}{4 V}\right) r$,

where $R$ and $V$ are the instantaneous radius and volume of the droplet, respectively. The above equation reflects the fact that the height-averaged velocity is determined by wetting and diffusion.

For dissolutive wetting, the Capillary number, $C a=\eta \dot{R} / \gamma$, is a function of the contact angle $(\theta)$, where $\gamma$ is the surface tension of the solvent and $\eta$ is the viscosity of the solvent.

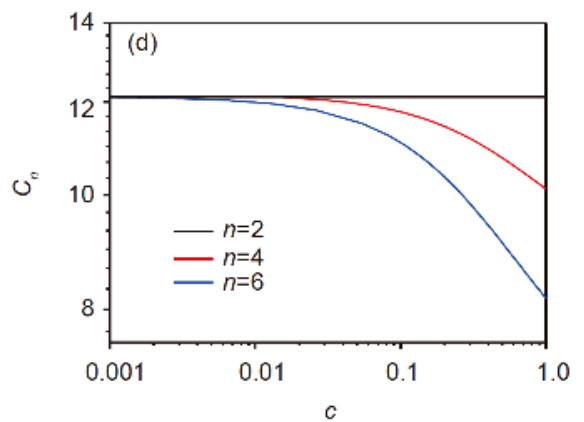

Figure 2 (Color online) (a) The height-averaged fluid velocity measured over $6 \mathrm{~s}$. The green triangles, red circles, and black squares are experiments with droplets on a glucose surface. The blue diamonds are the experimental results of droplets on PVA. The numbers represent the ratio of glycerol to water in the droplet. (b) The relation between the spreading velocity and contact angle is shown by a log-log plot. The experimental data can be expressed as $\dot{R} \sim \theta^{2.8}$. The open circles show the position of the turning point that divides the two stages of dissolutive wetting. (c) The relation between the spreading radius and time shown by a log-log plot. The turning points inside circles divide the process of dissolutive wetting into two stages. (d) The relation between the geometry parameter $C_{n}$ and the volume concentration $c$ shown by a $\log -\log$ plot. 
(a)
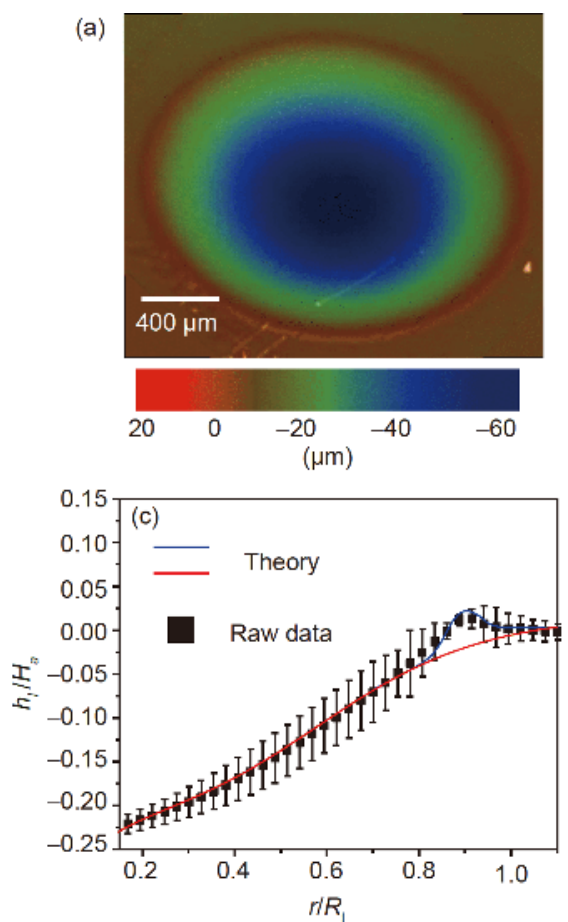

(b)
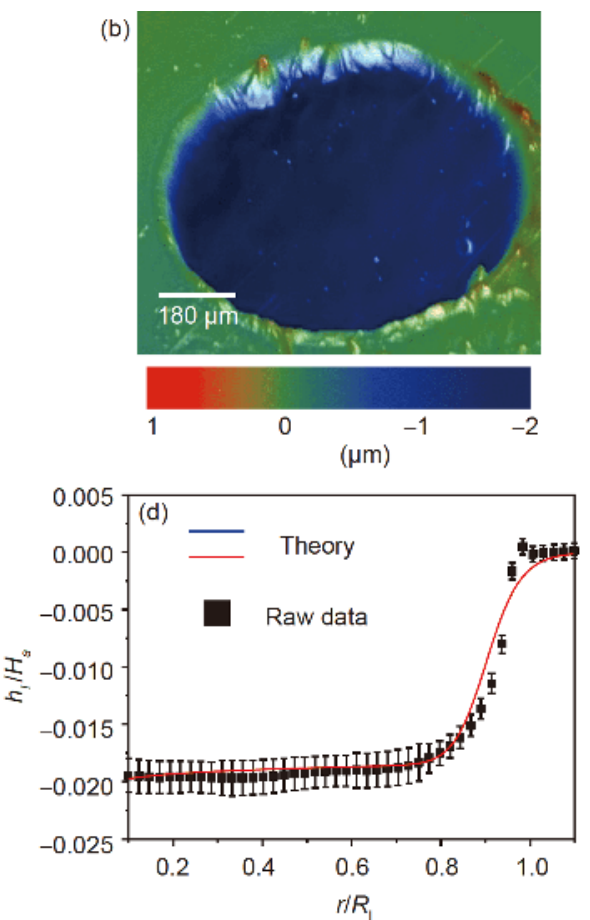

Figure 3 (Color online) The experimental and theoretical results for the solid-liquid interface shape. (a), (b) The shape of the solid-liquid interface measured via probe scanning. (c), (d) The shapes of the solid-liquid interfaces. The red lines are the theoretical results that only consider dissolution. The blue lines are the theoretical results that also consider evaporation. $R_{\mathrm{I}}$ is the radius where the droplet stops its first stage of spreading.

Usually, $C a<<1$ when a water droplet spreads on a solid substrate and the driving force of wetting is the surface tension. The relation between $C a$ and $\theta$ is as follows [25]:

$C a \propto \theta^{\chi}$.

The influence of the solute concentration on the viscosity is so small that a change in the viscosity during dissolutive wetting can be disregarded [26]. The surface tension near the contact line is nearly invariable because the concentration near the contact line reaches saturation quickly. Therefore, the scaling exponent $\chi$ can be obtained by fitting the experimental data of the wetting velocity $\dot{R}$ with respect to $\theta$ (see Figure 2).

We use a lubrication hypothesis to simplify the shape of the droplet shown in Figure 1 [27]. Usually, for a droplet on a hydrophilic surface, the height is generally smaller than the radius in dissolutive wetting. In addition, considering the small Bond number, the effect of gravity on the droplet shape can be ignored. The height profiles of the upper and lower droplets are given by a parabolic function and power function in cylindrical coordinates, respectively:

$\left\{\begin{array}{l}h_{\mathrm{u}}(r, t)=H_{\mathrm{u}}(t)\left[1-r^{2} / R^{2}(t)\right], \\ h_{1}(r, t)=H_{1}(t)\left[1-r^{n} / R^{n}(t)\right],\end{array}\right.$

$\left\{\begin{array}{l}H_{\mathrm{u}}(t)=\frac{1}{2} \theta_{\mathrm{u}}(t) R(t), \\ H_{1}(t)=\frac{2+n}{4 n} \theta_{1}(t) R(t),\end{array}\right.$

where $H_{\mathrm{u}}$ and $H_{1}$ are the upper and lower heights of the droplet at the origin, respectively. The air-liquid and solidliquid interfaces are described by different functions because the two interfaces are not symmetrical in most experiments. The assumptions about the interface shapes are concise and do not suffer from a loss of generality.

To derive the time evolution equation of the system, we use Onsager's variational principle. The Rayleighian is defined as:

$\Xi=\Phi+\dot{F}$,

where $\Phi$ is the energy dissipation of the system and $F$ is the potential energy. The time evolution equation of the system is determined by the condition that the Rayleighian is minimum with respect to $\dot{R}[23]$.

The system dissipation comprises the viscosity dissipation $\Phi_{\eta}$ and dissipation at the contact line $\Phi_{\xi}$, which is given as follows:

$\Phi=\Phi_{\eta}+\Phi_{\xi}$.

The viscosity dissipation is written as:

$$
\begin{aligned}
\Phi_{\eta} & =\int_{0}^{R} \frac{3 \eta}{2 h} u_{f}^{2} 2 \pi r \mathrm{~d} r \\
& =\frac{3 \pi^{2} \eta R^{4} C_{n}}{4 V}\left(\dot{R}-\frac{R \dot{V}}{4 V}\right)^{2},
\end{aligned}
$$

where $C_{n}$ is the shape factor of the liquid. We can obtain $C_{n}$ using theoretical and experimental methods (see Supporting Information). Some typical values of $C_{n}$ are shown in Figure 2. The other part of the dissipation function at the contact line 
is

$\Phi_{\xi}=\xi \pi R \dot{R}^{2}$,

where $\xi$ is the dissipation coefficient at the contact line.

The interfacial energy and chemical potential constitute the potential energy of the system. The energy of the interface is

$F_{\mathrm{s}}=\frac{\gamma_{\mathrm{SL}} \theta_{\mathrm{le}}^{2}+\gamma_{\mathrm{LV}} \theta_{\mathrm{ue}}^{2}}{2} \pi R^{2}+\frac{4 \gamma_{\mathrm{LV}}}{\pi R^{4}}\left(V_{\mathrm{u}}^{2}+\frac{n}{2} V_{1}^{2}\right)$,

where $\theta_{\mathrm{ue}}$ and $\theta_{\mathrm{le}}$ are the upper and lower equilibrium contact angle, respectively. Unlike nondissolutive wetting, when the solute molecules move into a droplet, they combine with the solvent molecules and form new structures [28]. When the dry solute diffuses into the liquid, the water molecules surround the solute [14], which results in a change in the interfacial energy of the solute. This induces a change in the free energy when the solute molecules separate from each other and combine with water molecules. Therefore, the separation and reconstruction of the solute molecules induce a change in free energy during the diffusion process. We simplify the relation between the chemical potential density and concentration into a linear form because only a little solute is dissolved $\left(\left(V_{\mathrm{l}} / V_{\mathrm{u}}\right)^{2}<<1\right)$. Therefore, the free energy density induced by the solute dissolving is

$f_{\mathrm{d}}=-\Gamma \phi$,

where $\Gamma=\left(k_{\mathrm{B}} T+S \Omega\right) N_{\mathrm{A}} / v_{\text {mole }}$ shows the change in the free energy induced by diffusion per unit concentration per unit volume, $v_{\text {mole }}$ is mole volume of the solute, $N_{\mathrm{A}}$ is the Avogadro number, $\Omega$ is the surface area of a solute molecule, $S$ is the spreading coefficient, $k_{\mathrm{B}}$ is the Boltzmann constant, $T$ is the temperature, and $\phi=V_{\mathrm{l}} / V$ is the volume concentration of the solute.

Using eqs. (7)-(10), we obtain the evolution equation of the system:

$\left(1+k_{\mathrm{cl}}\right) \dot{R}=\frac{k_{\mathrm{cl}} \gamma_{\mathrm{LV}} \theta_{\mathrm{ue}}^{2}}{2 \xi}\left(\frac{\theta_{\mathrm{u}}^{2}}{\theta_{\mathrm{ue}}^{2}}-1\right)+\left(\frac{3 k_{\mathrm{c}}^{2} \eta C_{n} \tau_{\mathrm{d}}}{8 \xi^{2} \pi} \Gamma+\frac{2}{3}\right) \frac{R \dot{V}}{V}$,

where $k_{\mathrm{cl}}=\xi \tilde{\theta} / 3 \eta C_{n}$ is used to compare the effects of $\Phi_{\eta}$ and $\Phi_{\xi} . \tilde{\theta}=n \theta_{1} / 2+\theta_{\mathrm{u}}$ and lower contact angle is much smaller than the upper contact angle; therefore, $\tilde{\theta}$ can be replaced by the contact angle $\theta$. Two terms on the right side of eq. (11) reflect the influence of the interface and dissolution energy on the droplet spreading. In our experiments, dissolutive wetting can be divided into two stages [17]. In the first stage, the droplet spreads quickly so that the upper contact angle is much larger than the upper equilibrium contact angle. The spreading velocity is much faster than the diffusion velocity and convective velocity; therefore, dissolution is disregarded. In the second stage, the spreading velocity decreases and the influence of dissolution becomes obvious. Therefore, there are differences between the wet- ting scaling exponents of the two stages, i.e., there is a turning point between the two stages (see Figure 2). In addition, in the second stage, the curve of the liquid-vapor interface changes slightly, i.e., the upper contact angle nearly equals the upper equilibrium contact angle $\left(\theta_{\mathrm{u}} \rightarrow \theta_{\mathrm{ue}}\right)$. Furthermore, mass transport primarily occurs in the second stage; accordingly, we focus on the second stage. In addition, the surface energy is less than the free energy induced by diffusion. Therefore, the first term on the right side of eq. (11) can be ignored. We further simplify eq. (11) such that

$\left(1+k_{\mathrm{cl}}\right) \dot{R}=\left(\frac{3 k_{\mathrm{cl}}^{2} \eta C_{n} \tau_{\mathrm{d}}}{8 \xi^{2} \pi} \Gamma+\frac{2}{3}\right) \frac{R \dot{V}}{V}$,

where $\tau_{\mathrm{d}}=V_{1} / \dot{V}$ is the characteristic time spent in dissolution and depends on the type of solute and solvent. The above equation shows that a linear relation can be established between the two self-similar processes in the second stage of dissolutive wetting. The above equation is solved as:

$$
\begin{aligned}
& \frac{R}{R_{0}}=\left(\frac{V}{V_{0}}\right)^{m}, \\
& m=\left(1+k_{\mathrm{cl}}\right)^{-1}\left(\frac{3 k_{\mathrm{cl}}^{2} \eta C_{n} \tau_{\mathrm{d}}}{8 \xi^{2} \pi} \Gamma+\frac{2}{3}\right) .
\end{aligned}
$$

The scaling $m$ shows that the evolution of the droplet radius depends on the change in free energy, extra friction of the contact line, and normal hydrodynamic friction in the second stage of dissolutive wetting. Considering eqs. (2) and (4), eq. (13) can be written as:

$R^{\frac{\chi(3 m-1)}{m}+1}=\omega^{-\frac{\chi}{m}}\left[\frac{\chi(3 m-1)}{m}+1\right] t$,

where $\omega=\operatorname{const} R_{0} V_{0}^{-m}\left(\eta / \gamma_{\mathrm{LV}}\right)^{\frac{m}{\chi}}$. The above equation is the governing equation of dissolutive wetting. For dissolutive wetting, the chemical potential, viscosity dissipation, and dissipation at the contact line determine the droplet spreading scaling. Considering eqs. (1) and (12), the height-averaged velocity can be written as:

$u_{\mathrm{f}}=\alpha \frac{r}{t}$,
$\alpha=\left(1-\frac{1}{4 m}\right)\left(\frac{m}{m+\chi(3 m-1)}\right)$,

where $\alpha$ represents the intensity of the convection in the droplet.

The evolution of the concentration field is described by the convection-diffusion equation as follows:

$\dot{c}+\mathbf{u} \cdot \nabla c=D \nabla^{2} c$,

where $\mathbf{u}=\left(u_{r}, u_{z}\right)$, and $u_{r}$ and $u_{z}$ are the velocities in the $r$ and $z$ directions, respectively. We can obtain the concentration field of dissolutive wetting by solving eq. (16). In most cases, the solute is primarily transported in the $r$ direction and the flow field can be expressed by the height-averaged velocity $u_{\mathrm{f}}$. Therefore, eq. (16) can be simplified such that 
$\frac{\partial c}{\partial t}+\frac{u_{\mathrm{f}} \partial c}{\partial r}=D\left(\frac{\partial^{2} c}{\partial r^{2}}+\frac{\partial c}{r \partial r}+\frac{c_{\mathrm{s}}-c}{H_{\mathrm{a}}^{2}}\right)$

The above equation is converted into a dimensionless form by introducing the following new variables:

$c^{\prime}=\frac{c}{c_{\mathrm{s}}}, t^{\prime}=\frac{t}{t_{\mathrm{d}}}, r^{\prime}=\frac{r}{R_{\mathrm{I}}}$,

where $R_{\mathrm{I}}$ is the radius wherein the droplet stops spreading in the first stage. Therefore, we have

$$
\begin{aligned}
& \frac{H_{\mathrm{a}}^{2} \partial c^{\prime}}{D \tau_{\mathrm{d}} \partial t^{\prime}}+\frac{P e}{A} \frac{\partial c^{\prime}}{\partial r^{\prime}} \\
& \quad=d^{-2}\left(\frac{\partial^{2} c^{\prime}}{\partial r^{\prime 2}}+\frac{\partial c^{\prime}}{r^{\prime} \partial r^{\prime}}\right)+1-c^{\prime},
\end{aligned}
$$

where $P e=u_{\mathrm{f}} R_{\mathrm{I}} / D$ and $d=R_{\mathrm{I}} / H_{\mathrm{a}}$. The $d^{-2}$ term can be neglected because the droplet is thin. We can further simplify eq. (19) such that

$\frac{\partial c^{\prime}}{u_{\mathrm{f}} \partial t^{\prime}}+\frac{\tau_{\mathrm{d}}}{R_{\mathrm{I}}} \frac{\partial c^{\prime}}{\partial r^{\prime}}=\frac{\tau_{\mathrm{d}} D\left(1-c^{\prime}\right)}{u_{\mathrm{f}} H_{\mathrm{a}}^{2}}$.

Solving the above equation, we obtain the evolution of the concentration field:

$c^{\prime}=1+\frac{\frac{c_{0}}{c_{\mathrm{s}}}-1}{\left.\mathrm{e}^{\Pi+\Psi}+\mathrm{e}^{\Pi+\left[(\beta / \alpha)^{2}+1\right.}\right] \Psi}$,

where $\Psi=\alpha(\zeta-\alpha \varsigma), \Pi=\tau_{\mathrm{d}} D \zeta / \alpha H_{\mathrm{a}}^{2}, \zeta=\ln r^{\prime}, \varsigma=\ln t^{\prime}, \beta=A \sqrt{B}$, $A=R_{\mathrm{I}} / H_{\mathrm{a}}$, and $B=\tau_{\mathrm{d}} D / H_{\mathrm{a}}^{2}$. In the above equation, the symbols with superscripts (') are dimensionless variables, and $c_{0}$ and $c_{\mathrm{s}}$ are the height-averaged concentration near the origin and saturation concentration, respectively. Their units are mass/volume. $\Psi=\alpha(\zeta-\alpha \varsigma)$ represents the influence of convection on the concentration distribution, and $\Pi=\tau_{\mathrm{d}} D \zeta / \alpha H_{\mathrm{a}}^{2}$ represents the influence of diffusion. $H_{\mathrm{a}}$ is the concentration boundary-layer thickness and $D$ is the diffusion coefficient. $B$ is the ratio of the dissolution time to the characteristic time of diffusion and indicates the diffusion intensity in the droplet, which is considerably larger than the

one in general and can be obtained by fitting the experimental data at the initial time. From the expression of $\Psi$, we can see that the solute transport along the radius depends on the characteristic convective velocity $\alpha R_{\mathrm{I}} / \tau_{\mathrm{d}}$. The process of solute transport is self-similarity with a scaling exponent of $\alpha$. The factor $\Psi$ shows that the concentration field evolution is similar to the transmission of a wave under the effect of convection. $\Pi$ depends on the ratio of the characteristic time of diffusion $\tau_{\mathrm{D}}=H_{\mathrm{a}}^{2} / \mathrm{D}$ to the characteristic time of dissolution $\tau_{\mathrm{d}} \cdot c_{0}$ is the height-averaged concentration at $r=0$, and $\beta$ indicates how sensitive the concentration distribution is to the convective velocity. According to eq. (21), the diffusive and convective effects are the key mechanisms that dominate the distribution of the concentration. Eq. (21) has two extreme solutions, and other solutions are a combination of these two solutions.

When the solute transported by convection is much stronger than that transported by diffusion, i.e., when $\Pi<<\Psi$, eq. (21) can be simplified such that

$$
c^{\prime}=1+\frac{\frac{c_{0}}{c_{s}}-1}{\mathrm{e}^{\Psi}+\mathrm{e}^{\left[(\beta / \alpha)^{2}+1\right] \Psi}} .
$$

Figure 4(a) shows the results of eq. (22). The solute transported by convection is considerably stronger than that transported by diffusion; therefore, the solute is transported to the contact line and accumulates at the rim of the sessile droplet. The concentration near the contact line keeps increasing until it reaches saturation. The height-averaged concentration decreases elsewhere away from the contact line. We call this solute transport mode the shifting mode.

When the effect of diffusion dominates the mode of the concentration distribution, i.e., $\Pi>>\Psi$, we can simplify eq. (21) such that

$$
c^{\prime}=1+\frac{\frac{c_{0}}{c_{\mathrm{s}}}-1}{\mathrm{e}^{\left.\Pi+\mathrm{e}^{\Pi+\left[(\beta / \alpha)^{2}+1\right.}\right] \Psi} .}
$$
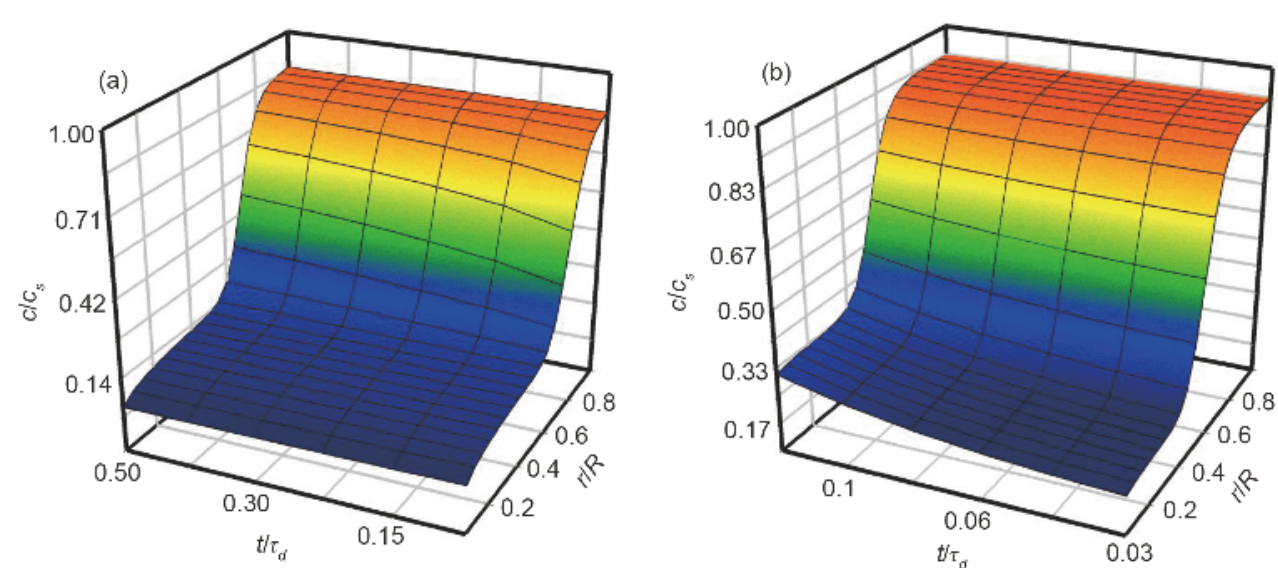

Figure 4 (Color online) The theoretical results of the concentration field evolution. (a) Calculated evolution of the concentration of the shifting mode. The parameters are $c_{0}=0.06 \mathrm{~g} \mathrm{~mL}^{-1}, \alpha=0.2, \tau_{\mathrm{d}}=13 \mathrm{~s}, R_{\mathrm{l}}=1.3 \mathrm{~mm}, B=165$, and $D=7.2 \times 10^{-4} \mathrm{~mm}^{2} \mathrm{~s}^{-1}$. (b) Calculated evolution of the concentration of the lifting mode. The parameters are $c_{0}=0.05 \mathrm{~g} \mathrm{~mL}^{-1}, \alpha=0.1, \tau_{\mathrm{d}}=60 \mathrm{~s}, R_{\mathrm{I}}=0.65 \mathrm{~mm}, B=250$, and $D=5.2 \times 10^{-5} \mathrm{~mm}^{2} \mathrm{~s}^{-1}$. 
The formation of the above equation is similar to the equation that ignores the influence of convection [18], which distinguishes it from the shifting mode, i.e., the lifting mode. The results can be explained such that the low surface tension gradient limits the height-averaged fluid velocity; therefore, diffusion is more obvious than convective transport.

We performed experiments to verify the existence of these two concentration evolution modes. The experimental results of Figures 5(a), (b) and (c), (d) agree with the shifting and lifting modes, respectively. The experiments for water dissolving glucose are shown in Figure 5(a) and (b). In this case, the diffusion coefficient of glucose to water is $7.2 \times$ $10^{-4} \mathrm{~mm}^{2} \mathrm{~s}^{-1}$. The surface tension of the solvent is approximately $6.9 \times 10^{-2} \mathrm{~N} \mathrm{~m}^{-1}$, viscosity of the liquid is approximately $0.8 \times 10^{-3} \mathrm{~Pa} \mathrm{~s}$, and characteristic length is approximately $1 \mathrm{~mm}$. Therefore, the dimensionless numbers are $P e \sim 100, C a \sim 10^{-6}, \alpha=0.2, \beta=25.69$, and $\Pi / \Psi \sim 0.01$. These results agree with the shifting mode. The concentration near the contact line increases quickly. On the contrary, the concentration around the center of the droplet remains low. Concentrations in the other regions decrease over time. Figure 5(b) shows that the higher the height-averaged concentration is the closer it is to the contact line. In addition, the saturation concentration region expands from the contact line to the center of the droplet. The experimental results for water dissolving PVA (Figure 4(c)) show that the transport direction of the solute is from bottom to top in the droplet. In this case, the diffusion coefficient of glucose to water is $5.2 \times$
$10^{-5} \mathrm{~mm}^{2} \mathrm{~s}^{-1}$, surface tension of the solvent is approximately $6.9 \times 10^{-2} \mathrm{~N} \mathrm{~m}^{-1}$, viscosity of the liquid is approximately $0.8 \times$ $10^{-3} \mathrm{~Pa} \mathrm{~s}$, and characteristic length is approximately $1 \mathrm{~mm}$. Therefore, $P e \sim 10, C a \sim 10^{-7}, \alpha=0.1, \beta=12.84$, and $\Pi / \Psi \sim 80$. The results verify the existence of the lifting mode. In the experiments, we also find different solid-liquid interface shapes after removing the droplet on the substrate surface. A qualitative perspective is that the saturation concentration region expands from the contact line to the center of the droplet. The depth around the center of the droplet is deeper than elsewhere (see Figure 3(a)). The shape of the solidliquid interface resembles a bowl. The experimental results for water dissolving PVA (see Figure 3(c)) show that the transport direction of the solute is from bottom to top in the droplet. The high concentration on the edge of the droplet suppresses the substrate from receding so that the shape of the solid-liquid interface resembles to that of a pan.

To examine the coupling effect of wetting and dissolution on the solid-liquid interface shape, we integrate Fick's first law over time to calculate the shape of the solid-liquid interface [29]:

$h_{1}=\frac{D}{\rho_{\mathrm{s}}} \int_{0}^{t} \frac{c_{\mathrm{s}}-c}{H_{\mathrm{a}}} \mathrm{d} t$.

When $t$ is much smaller than the characteristic time $\tau_{\mathrm{d}}$, the above equation can be simplified such that

$h_{1}\left(r_{0}, t\right)=\frac{\tau_{\mathrm{d}} D\left(c_{\mathrm{s}}-c_{0}\right)}{H_{\mathrm{a}} \rho_{\mathrm{s}} \beta_{1}}\left(\frac{t}{\tau_{\mathrm{d}}}\right)^{\beta_{1}}\left(\frac{r}{R_{I}}\right)^{-B}$,

where $\beta_{1}=\alpha^{2}+1$. The constant parameter $\tau_{\mathrm{d}} D\left(c_{\mathrm{s}}-c_{0}\right) /\left(H_{\mathrm{a}} \rho_{\mathrm{s}} \beta\right)$ is
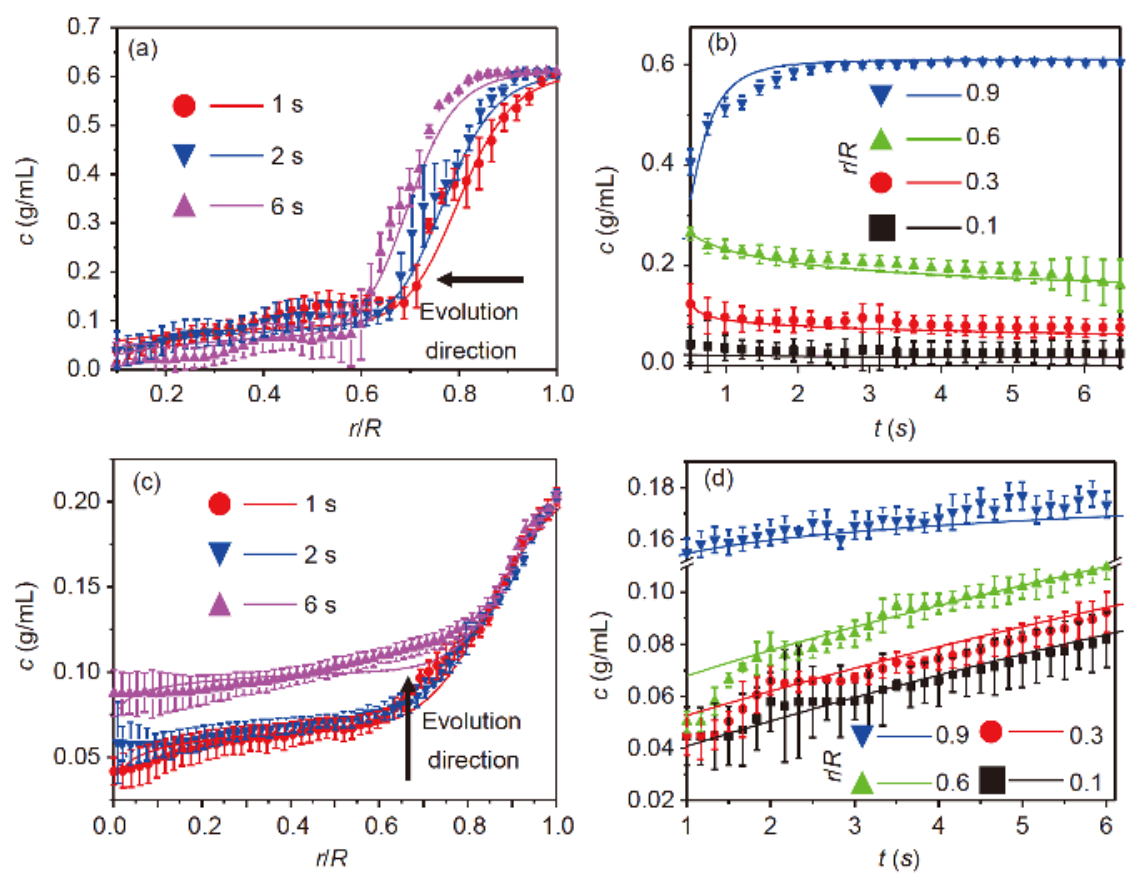

Figure 5 (Color online) The experimental and theoretical results of the concentration field and the solid-liquid interface shape. The graphs in the first row show the results for water dissolving glucose, and other graphs show the results for water dissolving PVA. (a), (c) The distributions of the concentration at different times. (b), (d) The evolutions of the concentration in different positions. 

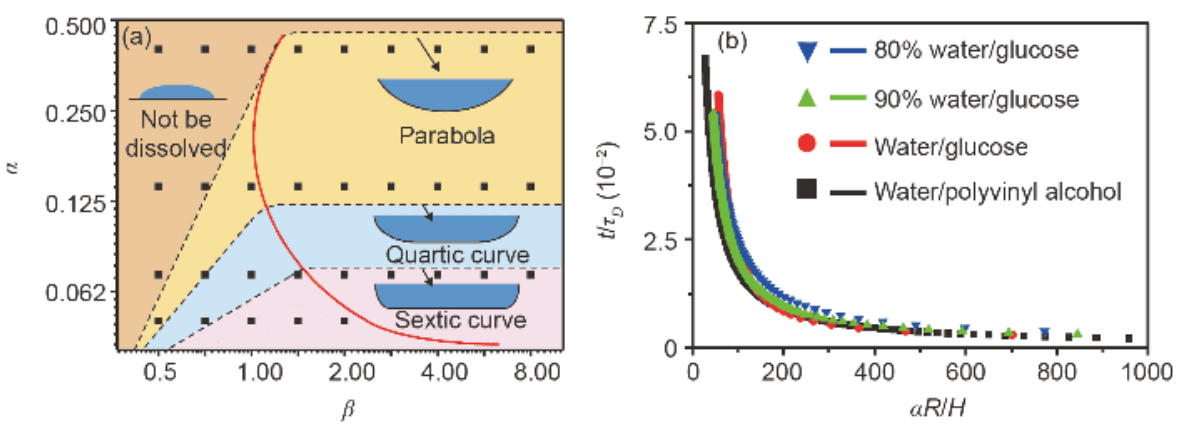

Figure 6 (Color online) (a) Phase diagram of the solid-liquid interface shape with respect to $\alpha$ and $\beta$. The dashed lines express where the interface shapes are parabolic, quartic curves, and sextic curves. (b) The relationship between the geometrical shape of the solid-liquid and dissolution time $\left(\beta^{2} \sim 10\right)$. $\tau_{\mathrm{D}}=H_{\mathrm{a}}^{2} / D$ and $R / H$ are the characteristic time of diffusion and the ratio of the spreading radius to the dissolution depth, respectively.

the characteristic depth of the hole. The depth of the hole has a scaling relation with time, and the scaling law is $\beta_{1}$, which considers the effects of convection and diffusion. When $t$ is close to the characteristic time $\tau_{\mathrm{d}}$, the above equation can be simplified such that

$h_{1}\left(r_{0}, t\right)=\frac{\tau_{\mathrm{d}} D\left(c_{\mathrm{s}}-c_{0}\right) \beta_{2}}{H_{\mathrm{a}} \rho_{\mathrm{s}}}\left(\frac{t}{\tau_{\mathrm{d}}}\right)^{\beta_{1}}\left(\frac{r}{R_{\mathrm{I}}}\right)^{-B}$,

where $\beta_{2}=\left(\beta_{1}+\alpha B\right)\left(t / \tau_{\mathrm{d}}\right)^{\beta^{2}} /\left[1+\left(r / R_{\mathrm{I}}\right)^{\beta^{2} / \alpha}\right]$. The influence of the solute deposition induced by convection on the solidliquid interface shape starts to appear. Eqs. (25) and (26) show that the physical parameters, except $\alpha$ and $\beta$, decide the amplitude of the function and are independent of the function type. The power function indicates that the depth of the hole decreases along the radial direction due to the competition of convection and diffusion. The shapes of the solid-liquid interfaces for different values of $\alpha$ and $\beta$ are shown in Figure 6(a). The solid-liquid interface shape can be described by power function. The powers of power functions negatively correlated with $\alpha$. The dashed lines express the interface shapes are parabola, quartic curves, sextic curves, respectively. The powers of the points between the dashed lines are fractional. For example, when $\alpha=0.25$ and $\beta=4$, the power value is 2.4 . The orange area shows where the shape change of the solid-liquid interface is slight enough to be ignored. With an increase in $\beta$, the shape of the solid-liquid interface is sensitive to $\alpha$, which indicates the convective intensity. When $\beta$ is sufficiently large (on the right side of the red line), the solid-liquid interface shape depends on the $\alpha$ and the influence of $\beta$ can be ignored in most cases. The red line in Figure 6(a) can be expressed as $\ln \beta=0.66(\ln \alpha) 2$ $+2.18 \ln \alpha+1.72$. Therefore, we can obtain the relation between the solid-liquid interface shape and dissolution time using a numerical method. For example, when water droplet dissolves the glucose surface, the parameters $\alpha$ and $\beta$ are 0.2 and 25.69, respectively. This point is on the right side of the solid red line in Figure 6(a); therefore, the solid-liquid shape depends on the parameter $\alpha$. Figure 6(b) shows the results of four dissolution couples. We find that the relations between the solid-liquid interface shape and dissolution time for the different couples are similar; therefore, we can obtain the formation time of the solid-liquid interface via the interface shape. When the diffusion coefficient is sufficiently small, the concentration boundary layer is very thin. Therefore, the effect of convection on the diffusion near the solid-liquid interface can be ignored.

\section{Conclusion}

In summary, we use Onsager's variational principle to obtain the evolution equation of dissolutive wetting, which shows that the height-averaged fluid velocity is proportional to the spreading velocity and inversely proportional to the spreading radius. Furthermore, we solved the convectiondiffusion equation based on the results of the height-averaged fluid velocity. The theoretical results indicate that the diffusive effect and convective effect determine the distribution of the concentration. The solute transport has two modes, namely the shifting and lifting modes. With the weakening of the convective effect, the mode of transport changes from shifting to lifting. We also find that the convective intensity $\alpha$ and parameter $\beta$ can be used to reflect the shape of the solid-liquid interface. We can inversely predict the solid-liquid interface according to the phase diagram, which illustrates the coupling influence of wetting and dissolution on the interface shape. With a decrease in the convective intensity, the solid-liquid interface shape more closely resembles a pan. Our theory brings a physical image to the problem, together with a detailed explanation.

\section{Supporting Information}

The supporting information is available online at phys.scichina.com and link.springer.com. The supporting materials are published as submitted, without typesetting or editing. The responsibility for scientific accuracy and content remains entirely with the authors.

This work was supported by the National Natural Science Foundation of China (Grant Nos. 11722223, 11672300, 11872363, and 51861145314), the 
Chinese Academy of Sciences (CAS) Key Research Program of Frontier Sciences (Grant No. QYZDJ-SSW-JSC019), the Strategic Priority Research Program of the Chinese Academy of Sciences (Grant No. XDB22040401), and the CAS Interdisciplinary Innovation Team Project.

1 W. Yang, H. T. Wang, T. F. Li, and S. X. Qu, Sci. China-Phys. Mech. Astron. 62, 014601 (2018).

2 Z. P. Xu, and Q. S. Zheng, Sci. China-Phys. Mech. Astron. 61, 074601 (2018).

3 E. Mohtarami, A. Baghbanan, M. Eftekhari, and H. Hashemolhosseini, Theor. Appl. Fract. Mech. 89, 110 (2017).

4 A. M. Hynes, H. Ashraf, J. K. Bhardwaj, J. Hopkins, I. Johnston, and J. N. Shepherd, Sens. Actuat. A-Phys. 74, 13 (1999).

5 S. Biswas, J. Doherty, D. Saladukha, Q. Ramasse, D. Majumdar, M. Upmanyu, A. Singha, T. Ochalski, M. A. Morris, and J. D. Holmes, Nat. Commun. 7, 11405 (2016).

6 B. J. Carey, J. Z. Ou, R. M. Clark, K. J. Berean, A. Zavabeti, A. S. R. Chesman, S. P. Russo, D. W. M. Lau, Z. Q. Xu, Q. Bao, O. Kevehei, B. C. Gibson, M. D. Dickey, R. B. Kaner, T. Daeneke, and K. Kalantar-Zadeh, Nat. Commun. 8, 14482 (2017).

7 J. B. Dressman, G. L. Amidon, C. Reppas, and V. P. Shah, Pharmaceut. Res. 15, 11 (1998).

8 L. Yin, B. Murray, and T. Singler, Acta Mater. 54, 3561 (2006).

9 R. Hellmann, S. Cotte, E. Cadel, S. Malladi, L. S. Karlsson, S. Lozano-Perez, M. Cabié, and A. Seyeux, Nat. Mater. 14, 307 (2015).

10 J. A. Hyatt, and P. M. Jacobs, Geomorphology 17, 305 (1996).

11 W. F. Zhou, Eng. Geol. 31, 50 (1997).

12 L. Ristroph, J. Fluid Mech. 838, 1 (2018).
13 C. Cohen, M. Berhanu, J. Derr, and S. Courrech du Pont, Phys. Rev. Fluids 1, 050508 (2016).

14 Q. Z. Yuan, and Y. P. Zhao, Phys. Rev. Lett. 104, 246101 (2010).

15 Y. P. Zhao, Physical Mechanics of Surfaces and Interfaces (Science Press, Beijing, 2012).

16 D. Wheeler, J. A. Warren, and W. J. Boettinger, Phys. Rev. E 82, 051601 (2010), arXiv: 1006.4881.

17 E. Saiz, M. Benhassine, J. De Coninck, and A. P. Tomsia, Scripta Mater. 62, 934 (2010).

18 J. Yang, Q. Yuan, and Y. P. Zhao, Int. J. Heat Mass Transfer 118, 201 (2018).

19 P. Protsenko, O. Kozlova, R. Voytovych, and N. Eustathopoulos, J. Mater. Sci. 43, 5669 (2008).

20 O. Kozlova, R. Voytovych, P. Protsenko, and N. Eustathopoulos, J. Mater. Sci. 45, 2099 (2009).

21 N. Alleborn, and H. Raszillier, Chem. Eng. Sci. 59, 2071 (2004).

22 P. Protsenko, J. P. Garandet, R. Voytovych, and N. Eustathopoulos, Acta Mater. 58, 6565 (2010).

23 J. Chapuis, E. Romero, F. Soulié, C. Bordreuil, and G. Fras, Heat Mass Transfer 52, 2283 (2015).

24 X. Man, and M. Doi, Phys. Rev. Lett. 116, 066101 (2016), arXiv: 1602.04891 .

25 P. G. de Gennes, Rev. Mod. Phys. 57, 827 (1985).

26 V. Stanek, and J. Szekely, Chem. Eng. Sci. 25, 699 (1970).

27 J. R. Lister, G. G. Peng, and J. A. Neufeld, Phys. Rev. Lett. 111, 154501 (2013), arXiv: 1310.0484.

28 Q. Z. Yuan, J. H. Yang, Y. Sui, and Y. P. Zhao, Langmuir 33, 6464 (2017).

29 X. H. Wang, W. H. Shen, X. F. Huang, J. L. Zang, and Y. P. Zhao, Sci. China-Phys. Mech. Astron. 60, 064612 (2017). 